\title{
Cambios en el control de la salud en el adolescente estudiante
}

\author{
N. Zdanowicz, MD* \\ P. Janne, PD** \\ CH. Reynaert, MD***
}

* Co-Jefe del Servicio de la Unidad de

Psicosomática, Psiquiatra Director de la

Clínica para Adolescentes Universidad

Católica de Lovaina

Clínica de Mont-Godinne, 5530 Yvoir

E-mail: nicolas.zdanowicz@pscl.ud.ac.be

** Profesor de Psicología. Universidad

Católica de Lovaina

Clínica de Mont-Godinne, 5530 Yvoir

E-mail: janne@clap.ucl.ac.be

*** Profesor de Psiquiatría, Jefe del

Departamento, Director de la Unidad de

Psicosomática. Universidad Católica de

Lovaina, Clínica de Mont-Godinne, 5530 Yvoir

E-mail: christine.reynaert@psme.ucl.ac.be

BÉLGICA

RESUMEN - Objetivos: Examinar los cambios en la escala Multidimensional de Asignación del Control de la Salud (MHLC) durante la adolescencia una muestra de estudiantes de edades comprendidas entre los 13 y los 25 años.

Método: 814 estudiantes cumplimentaron el cuestionario MHLC de Wallston.

Resultados: Aunque no se observaron cambios en función de la edad en la sub-escala de Asignación Interna del Control de la Salud (IHLC), sí se produjo, sin embargo, una disminución en la Asignación del Control de la Salud al Poder de terceros (PHLC) con un aumento en conjunto de la proporción "interno" frente a "externo". El sexo de los sujetos no mostró efectos significativos sobre estos cambios.

Conclusiones : La adolescencia no parece estar marcada por un incremento en el sentimiento de autocontrol sino más bien por una "desresponsabilización" (retirada de la responsabilidad) de los padres. 


\section{Introducción}

En 1978, K.A. Wallston y sus colegas desarrollaron la escala multidimensional de asignación del control de la salud (MHLC) como un método de investigación de la opinión de una persona en cuanto al grado de responsabilidad que dicha persona mantiene en la determinación de su estado de salud. Esta escala es multidimensional en el sentido de que hace posible diferenciar entre tres tipos distintos de opiniones: dos "externas" y una "interna". Los individuos con un control interno opinan que el grado de perfecta salud que disfrutan depende de su comportamiento personal. Esta propensión se mide mediante la sub-escala de asignación interna del control de la salud (IHLC). Los individuos con control externo opinan que, o bien están sujetos a la suerte y el destino, parámetro medido por la sub-escala de asignación al "azar" del control de la salud (CHLC), o bien a la acción de terceros: esta es la sub-escala de asignación del control de la salud al "poder de terceros" (PHLC).

La cuantiosa literatura generada por esta escala, incluyendo una crítica realizada por A. Pauwels (1999) ha demostrado que la MHLC es una buena herramienta de pronóstico del estado de salud tanto en medicina como en psiquiatría, en especial, mediante el estudio de las actitudes primaria y secundaria de los individuos. Norman y Bennett (1996) mostraban que la IHLC estaba asociada con conductas saludables -segurasmientras que la PHLC se asociaba más con conductas poco saludables, de riesgo. En cuanto a la CHLC, esta parece indicar un sentimiento de pérdida de control.

Por lo que nosotros sabemos, tan sólo dos estudios realizados en este campo han aplicado la MHLC al estudio de adolescentes. El estudio realizado por W.R. Stanton
(1995) se ocupó de la utilidad y estabilidad de la MHLC en la adolescencia. Además de confirmar la utilidad de la MHLC, mostraba diferencias entre los sexos en la asignación del control. El mencionado autor halló variaciones en la CHLC y la PHLC en edades comprendidas entre los 13 y los 15 años, pero sólo entre las adolescentes. El segundo estudio, realizado por S. Nada-Raja (1994), analizó un grupo de más de 800 jóvenes, de 15 años de edad. Este estudio no se ocupaba de la influencia de la edad en la MHLC pero evidenciaba diferencias por sexo en las puntuaciones medias de la IHLC y la PHLC. También revelaba la influencia de los acontecimientos vitales adversos y de las opiniones maternas en relación con la asignación del control en la formación de un nivel bajo o elevado de IHLC entre sus hijas. Los niveles altos de la IHLC entre los sujetos del sexo masculino mantenían correlación con niveles altos de respaldo social, relaciones de cariño o percepción subjetiva de fortaleza física. Sin embargo, el mencionado autor exponía como conclusión que se obtenía un beneficio escaso de la utilización de la MHLC en niños jóvenes debido a que no logró hallar ninguna correlación entre el nivel de salud física o mental y la MHLC. Sin embargo, debería hacerse constar que la población de este estudio estaba formada por un grupo de sujetos jóvenes que como era de suponer no padecían enfermedades.

La posibilidad de que la MHLC varíe según la edad, nos parece de importancia suficiente como para merecer atención adicional. Si tomamos como ejemplo el tema de los trasplantes en niños, M.L. Stubher (1993) llegó a afirmar que la implicación de un niño en un programa de trasplante depende en un primer momento de los padres, pero que podría plantearse cuándo y cómo, al llegar a adolescente, éste lo asume como responsabilidad propia. Es razonable 
esperar que la comprensión de la forma en que la MHLC cambia con la edad proporcione (al menos en parte) respuesta a esta pregunta (Zdanowicz, Reynaert et al. 1996 y Zdanowicz, Janne et al. 1998 a), pero que además también aporte distintos indicadores sobre diferentes sectores de patología juvenil como:

- Alteración en el desarrollo psicológico de los jóvenes que padecen una enfermedad crónica entre la niñez y la adolescencia tal como se ha mostrado en la diabetes en adultos (Reynaert, Janne et al. 1995a).

- Evaluación psicológica de los jóvenes con conductas de riesgo tales como las que se han demostrado en adultos alcohólicos (Zdanowicz, Reynaert et al. 1998b).

- Desarrollo de campañas de prevención para jóvenes.

Estamos convencidos de que entre la infancia y la madurez debe producirse una reestructuración de la experiencia vivida sobre el control y la posesión de la salud, con el relevo de los padres y profesores a los adolescentes. Basándonos en la información aportada por Stanton, estaríamos en condiciones de comprobar la hipótesis relacionada con el debilitamiento tanto de la PHLC como de la CHLC, así como con las diferencias por el sexo. En ese caso sería interesante extender el intervalo de edad a las comprendidas entre los 12 y los 25 años para de esta forma incluir también a aquellos individuos que viven bajo las condiciones que se describen como "adolescencia prolongada" (Organización Mundial de la Salud -WHO-, 1994). Esta es la razón por la que consideramos interesante la idea de incluir a estudiantes universitarios ya que sus circunstancias cumplían los criterios de "adolescencia prolongada”. Además, exponíamos la hipótesis de un incremento en la IHLC. Esta suposición está documentada en numerosos estudios previos sobre "los procesos de reestructuración de la adolescencia" que llevan a apreciar un aumento en el sentido de responsabilidad durante la adolescencia, y por ello igualmente en un sentido de control sobre el entorno personal general (Slomowitz M. 1991). Estos hallazgos explican también nuestra hipótesis de un incremento en la ratio I/E (tal como se define por Ch. Reynaert, Janne et al. 1995 b) durante la adolescencia.

- 1. ${ }^{\text {a }}$ hipótesis: un incremento de la IHLC,

- 2. a hipótesis: una disminución de la PHLC,

- 3. ${ }^{\text {a }}$ hipótesis: un incremento de la ratio interno/externo.

- 4. ${ }^{a}$ hipótesis: una disminución de la CHLC.

\section{Método}

Este estudio se llevó a cabo en el mes de junio de 1999. Aparte de la MHLC, los sujetos describieron a sus familias utilizando la $3^{a}$ edición (Olson \& Killorin 1985) del Cuestionario Circumplex de Olson (Olson, Sprenkle et al. 1973). Esta evaluación adicional sirvió para proporcionar un medio de correlacionar la MHLC y las relaciones familiares en un futuro estudio si nuestras hipótesis se demuestran correctas. En efecto, diversos estudios también relacionaron las interacciones familiares con el estado de salud de los jóvenes. Por lo tanto, tras haber llevado a cabo una investigación sobre la correlación entre la MHLC y la edad juvenil, nos pareció lógico investigar la correlación entre sus actitudes sobre su salud y la naturaleza de sus dinámicas familiares. Hicimos uso de las versiones en francés de 
la MHLC (Janne 1989) y del cuestionario de Olson (Fontaine 1985).

Wallston (1987) al igual que Stanton mostraron que la consistencia interna de cada sub-escala de la MHLC parecía adecuada (entre 0,67 y 0,77 ) y presentaba poca interdependencia entre las tres combinaciones de sub-escalas. Hemos comprobado dicha independencia de sub-escalas y, como hiciera Wallston, hallamos que las sub-escalas IHLC y PHLC son estadísticamente independientes $(0,087)$, que las sub-escalas IHLC y CHLC presentan una ligera correlación negativa $(-0,301)$ y que las sub-escalas PHLC y CHLC presentan una ligera correlación positiva $(0,226)$.

\section{Participantes y procedimiento}

El grupo de estudio se constituyó de dos formas distintas cada método, formándo un subgrupo. Se utilizó este procedimiento para obtener categorías diferentes de edad y de adolescentes con diferentes puntos de vista. El primer subgrupo "escolar" estaba compuesto por jóvenes reclutados de cada uno de los 6 cursos de educación general secundaria (es decir, normalmente entre los 12 y los 18 años) en tres Centros de Secundaria diferentes pertenecientes a la región de Namur (Bélgica). Pedimos a los jóvenes, en presencia de un psiquiatra examinador, que facilitaran información demográfica (edad, nombre, sexo, curso, nacionalidad) y que cumplimentaran la escala MHLC y la de Olson sobre su familia real y sobre su familia ideal. El segundo subgrupo de estudiantes de la "UCL" estaba formado por estudiantes universitarios de segundo año de Licenciatura en Psicología en la Universidad Católica de Lovaina (Catholic University of Louvain UCL) que reclutaron a otros jóvenes conocidos suyos, y que tenían que ser:
- De edades comprendidas entre los 13 y $\operatorname{los} 25$ años

- Solteros o viviendo como pareja no casada

- Desempleados, sin una carrera profesional y/o estudiantes.

Estos deben cumplimentar un cuestionario anónimo que incluía la fecha de nacimiento, su sexo, la escala MHLC y la escala Olson.

Los tres criterios arriba descritos son los propuestos por la Organización Mundial de la Salud (WHO 1994) como determinantes de la "situación del adolescente". Para conseguir que la población de nuestro estudio fuera aun más homogénea, se exigió como requisito que fueran: estudiantes, caucásicos y francófonos.

Se asume por defecto que la población de nuestro estudio está "sana". De hecho, puesto que la población no se buscó en un centro de salud o en un hospital, no podemos excluir la posibilidad de que uno o más de estos jóvenes hubieran podido ser hospitalizados por alguna razón que nosotros desconociéramos.

\section{Análisis y presentación de resultados}

Los análisis estadísticos se llevaron a cabo utilizando el progama SPSS para Windows 95/98/NT para Modelos Avanzados versión 9.0S. Considerando el elevado número de sujetos y la necesidad de analizar la influencia de diversas co-variables, utilizamos tests paramétricos. Las variables cuantitativas fueron comparadas utilizando el test-t (Student's t-test). Se consideró como probable el ratio $\mathrm{F}$ en los valores 0,05 $<\mathrm{p}>0,01$, y como muy probable en $\mathrm{p}<$ 0,01 . El test de Pearson $\chi^{2}$ se utilizó para 
comparar proporciones con los mismos límites p. Las correlaciones entre las variables continuas se evaluaron utilizando el coeficiente de Pearson, si era necesario verificarlas para una co-variable (correlación parcial). Todas las estadísticas son pareadas.

Los resultados se presentan de la siguiente forma: análisis de parámetros demográficos (edad y sexo), discusión del impacto de los parámetros demográficos, análisis paso a paso de la IHLC, ratio I/E en la PHLC y CHLC con relación a la edad y verificado, si era necesario, por sexo.

Otros parámetros demográficos convencionales como el origen étnico, la ocupación y el nivel de estudios no son relevantes para este estudio. El origen étnico y la ocupación estaban predeterminados por los criterios de selección y el nivel de estudios como consecuencia del diseño de este estudio es dependiente de la edad. Por supuesto éramos conscientes de que la situación socioeconómica de los sujetos de nuestro estudio no era representativa de la población media de Bélgica. Aunque en Bélgica la escolarización hasta los 18 años es obligatoria y gratuita y por tanto ello implia cierto grado de heterogeneidad en la enseñanza superior, éste no es el caso con respecto a los estudiantes universitarios, y por ello tampoco lo es en nuestra muestra de estudiantes de la Universidad UCL. A pesar de que el Ministerio de Educación belga establece el registro y las tasas de inscripción para, en teoría, permitir a todos el acceso gratuito a los estudios superiores, la mayoría de los estudiantes universitarios per- tenecen a la clase media. Sin embargo, para el objeto del presente estudio, consideramos que, en la medida en que esta situación mantiene a estos jóvenes bajo la dependencia financiera de sus padres con independencia de la edad que tengan, constituye ello una ventaja más que un inconveniente. Por otra parte, esta falta de representatividad limita la generalización de nuestras observaciones a la totalidad de los adolescentes.

\section{Resultados}

\section{Características demográficas}

\section{Distribución por edad}

La totalidad de la muestra agrupa a 810 sujetos con una edad media $(\mathrm{SD} \pm 3)$ de 18 años (13 a 25). El test-t de Student mostraba una tendencia hacia edades medias distintas en los dos subgrupos $(t=-28.594 \mathrm{p}=0,000)$. El subgrupo "escolar" era más joven, con una edad media de 15,3 $( \pm 1,8)$; el subgrupo "UCL" presentaba una edad media de 19,7 $( \pm 2,2)$. Este dato era previsible puesto que era lo más probable que los estudiantes universitarios pidieran a otros estudiantes también universitarios que cumplimentaran el cuestionario, es decir, estudiantes pertenecientes al mismo intervalo de edad.

\section{Distribución según el sexo}

Aunque la proporción por sexos era de 328 hombres frente a 434 mujeres, es decir se daba una razón de 0,75 , se presentaba

Tabla I

Comparación de edades en los subgrupos

\begin{tabular}{lccc} 
& $\mathrm{N}$ & Media (años) & $\mathrm{SD}$ \\
\hline «Escuela» & 301 & 15,3 & 1,8 \\
«UCL» & 489 & 19,7 & 2,2 \\
Total & 790 & 18,0 & 3,0 \\
\hline
\end{tabular}


una diferencia según sexo en la distribución de los individuos entre los subgrupos (tabla III). Esta diferencia se mostraba estadísticamente probable $\left(\chi^{2}\right.$ de Pearson $=63,259, \mathrm{p}$
$=0,000)$. A pesar de que el subgrupo "escolar" estaba constituido por un $60 \%$ de hombres, el subgrupo "UCL" estaba intregrado en más del $60 \%$ por mujeres.

Tabla II

Distribución por sexos en los subgrupos

\begin{tabular}{lcccc} 
& Chicas & Chicas & Chicos & Chicos \\
& $\mathrm{N}$ & $\%$ & $\mathrm{~N}$ & $\%$ \\
\hline «Escuela» & 105 & 38 & 171 & 62 \\
«UCL» & 331 & 67,7 & 158 & 32,3 \\
Total & 436 & 53,6 & 329 & 40,4 \\
\hline
\end{tabular}

Impacto de los parámetros demográficos

Considerando las diferencias significativas en la variable "edad" entre los subgrupos, era imprescindible recoger este parámetro al comparar los subgrupos en el análisis estadístico. Sin embargo, debido a que nuestras hipótesis de relación se habían formulado con relación a la edad, este parámetro se verificaba desde el resultado. Es más no estábamos interesados en las diferencias entre subgrupos, ya que estos individuos formaban parte de una única muestra global de adolescentes "sanos", por lo que la comparación entre subgrupos era innecesaria. El mismo razonamiento podía aplicarse a la diferencia entre subgrupos en cuanto a la distribución por sexo. Sin embargo, debía tomarse en consideración que los estudios previos habían mostrado diferencias en los valores de la MHLC entre chicos y chicas, por lo que el factor "sexo" debería comprobarse.

\section{Comprobación de las hipótesis}

\section{$1^{a}$ hipótesis: Incremento en la IHLC con la edad}

A pesar de la hipótesis sobre un incremento en la IHLC en relación con la edad durante la adolescencia, este incremento era muy reducido para ser estadísticamente significativo: el coeficiente de Pearson era 0,030 y el nivel de probabilidad $p$ de 0,409 ( $\mathrm{N}=760)$ no era significativo. Si esta hipótesis se somete a reconsideración al tener en cuenta el sexo, los resultados continúan siendo estadísticamente no significativos ( $\mathrm{p}$ $=0,230 ; \mathrm{r}=0,0448)$.

Los chicos habían obtenido puntuaciones mayores que las chicas en 0,8009 puntos (media en las chicas $=23,7786, \mathrm{SD}=$ 4,7677 y media en los chicos $=24,5795$, SD = 4,9259). Un análisis estadístico, distinto para chicos y para chicas, sobre los cambios en la IHLC en relación con la edad, no consiguió tampoco revelar ninguna correlación (chicos: $r=0,02, p=0,726$; chicas: $r=$ $0,065, \mathrm{p}=0,182)$.

$2^{a}$ hipótesis: Disminución en la PHLC con la edad

De hecho, se observó una disminución en la PHLC según la edad. El coeficiente $r$ de Pearson para esta correlación fue de $-0,176$ $\mathrm{y}$ el valor de $\mathrm{p}$ fue inferior a 0,001 $(\mathrm{N}=$ 762). El coeficiente de correlación parcial comprobado según el sexo fue de r -0,1675 con el mismo nivel de probabilidad. 


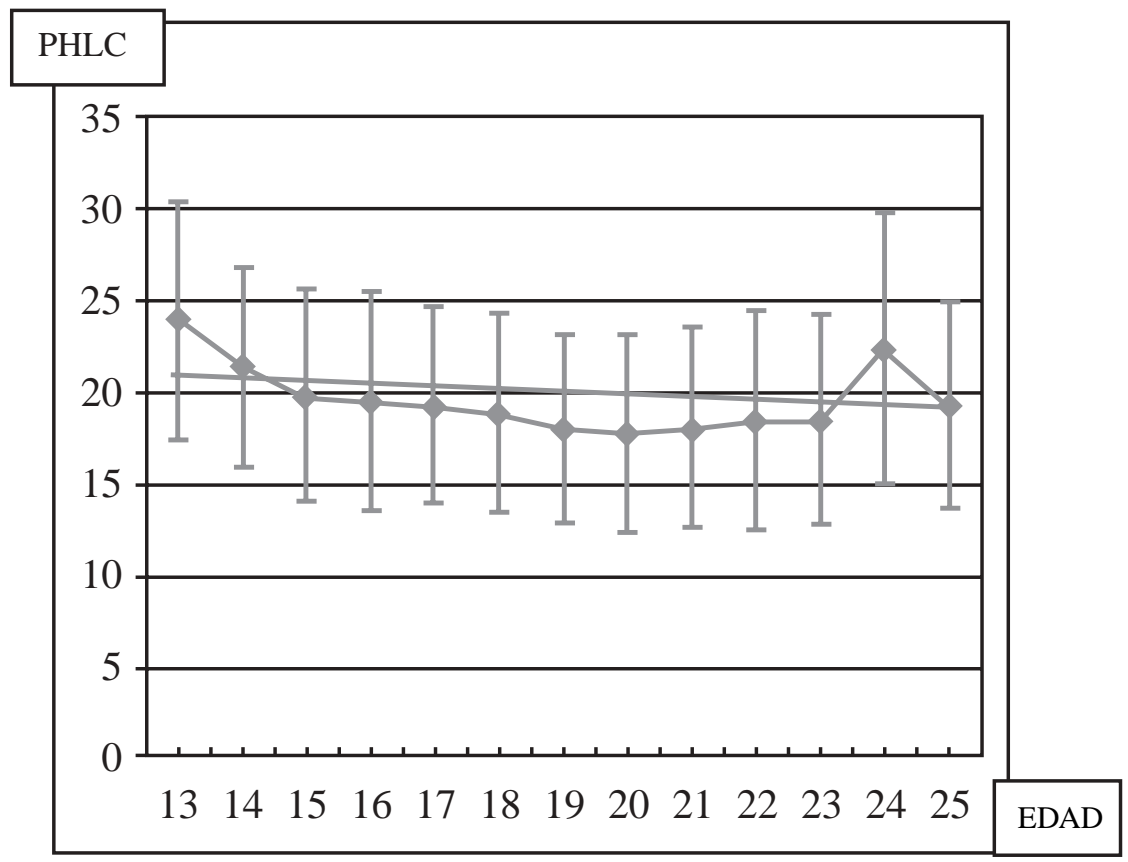

Figura 1. Medias en sujetos “sanos” en relación con la edad (años).

Un análisis estadístico, distinto para chicos y para chicas, de los cambios en la PHLC en relación con la edad, confirmó la correlación (chicos: $\mathrm{r}=-0,160, \mathrm{p}=0,05$; chicas: $r=-0,173, p<0,001)$. Como se refleja en la figura 1, no se aprecia "linearidad" en la zona especialmente próxima al final de la curva. Avanzamos dos razones para ello:

1. O los pocos sujetos de edades comprendidas entre los 24 y los 25 años podrían haber producido un sesgo en la estadística ya que desgraciadamente, estos sujetos presentaron una puntuación más alta que la esperada.

2. O, que dado que el porcentaje de la varianza explicada es baja, otro factor podría haber intervenido: el graduarse en la Universidad y encontrarse más cerca de acceder al mundo adulto, podría haber modificado las expectativas que tendrían estos sujetos de entre 24 y 25 años con respecto a terceros.

\section{$3^{a}$ hipótesis: Incremento de la proporción} $I / E$

Se presentaba una tendencia estadística hacia un incremento en el factor "interno" (figura 2) en relación con la edad, que estaba en línea con nuestras hipótesis. El coeficiente $\mathrm{r}$ de Pearson fue de 0,078 con una tendencia en $\mathrm{p}=0,035(\mathrm{~N}=736)$. El coeficiente de correlación parcial verificado por sexo se mantuvo significativo en 0,0835 con un nivel $\mathrm{p}=0,027$.

Un análisis estadístico diferente para chicos y chicas no consiguió revelar tampoco ninguna correlación (chicos: $\mathrm{r}=0,086, \mathrm{p}=$ 0,147; chicas: $r=0,081, p=0,081$ ).

\section{$4^{a}$ hipótesis: Descenso en la CHLC con la edad}

En contradicción con nuestra hipótesis, el análisis estadístico mostró que la CHLC ten- 


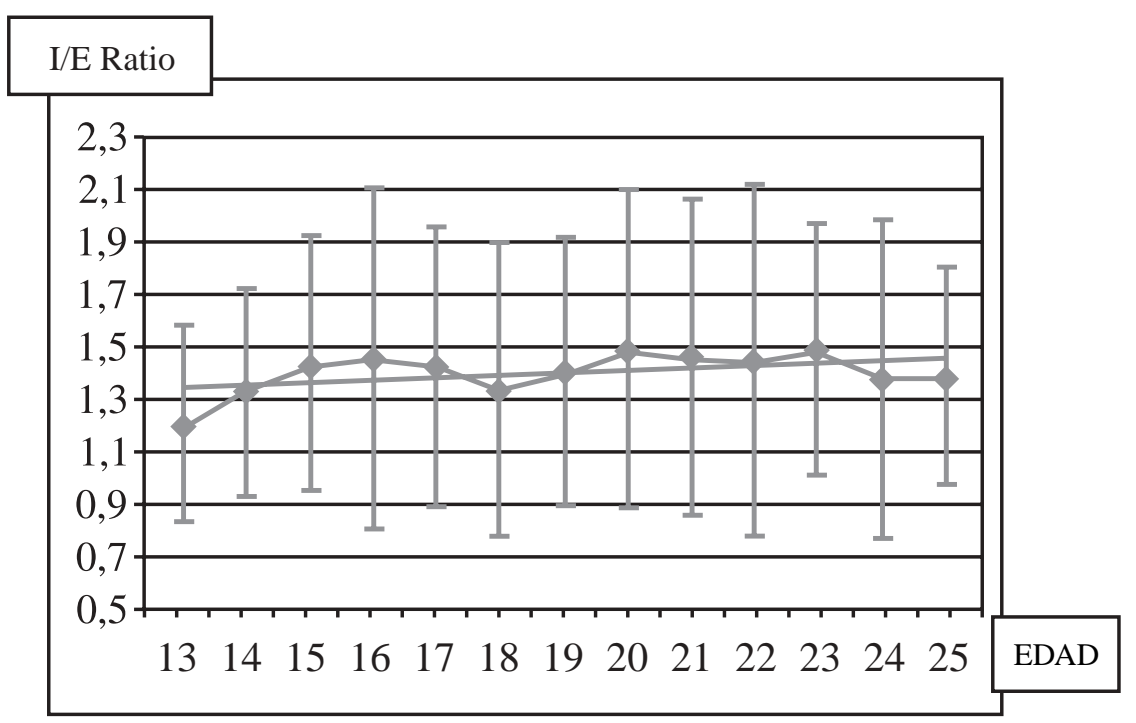

Figura 2. Medias I/E ratio en sujetos “sanos” en relación con la edad (años).

día a incrementarse durante la adolescencia: el valor $\mathrm{r}$ de Pearson fue de 0,061 y la p fue de 0,092. Esta correlación no se confirmó con una correlación de Pearson comparada por sexo $(r=0,049, p=0,188)$ y el análisis estadístico separado para chicos y chicas no consiguió revelar ninguna correlación (chicos: $\mathrm{r}=$ 0,051, $\mathrm{p}=0,382$; chicas: $\mathrm{r}=0,047, \mathrm{p}=0,328$ ).

\section{Discusión}

Como puede apreciarse, mientras algunas de nuestras hipótesis se confirman, debe tenerse en cuenta que estos hallazgos pueden aplicarse únicamente a una pequeña parte de la varianza. Esto no obstante es tranquilizador. De hecho habría resultado sorprendente que los cambios en un parámetro tan importante como es el sentimiento de responsabilidad sobre la propia salud hubieran dependido exclusivamente de la edad. Otra serie de factores debían de estar implicados, tales como la actitud de los padres frente a la salud, el entorno sociocultural, e incluso, como se mostraba en el estudio de Nada-Raja (1994), los acontecimientos vitales o factores constitucionales, tales como la fortaleza física en los chicos.

Aparentemente podrían identificarse tres factores que apoyarían o incluso irían más allá de las observaciones realizadas por Stanton. Mientras que en las chicas, en relación con la edad, se han apreciado cambios en la PHLC y en la CHLC, nuestro estudio muestra que también estos cambios aparecen en los chicos:

1. El sentimiento de responsabilidad con respecto a la propia salud en la adolescencia no cambia mucho mediante un incremento en el propio parámetro (IHLC) sino más bien a través de una disminución en el sentimiento de que los otros son los responsables de la salud propia. El sentimiento personal de responsabilidad se forma en un momento temprano, y ya en la adolescencia, tiene valores similares a los que se encuentran en 
una población de control de mayor edad. De hecho, si comparamos el nivel medio de la IHLC (IHLC 24,1, SD 4,7, N = 782) en la población de nuestro estudio con la muestra control compuesta por adultos del mismo país (IHLC 22, $7 \mathrm{SD} 7,3, \mathrm{~N}=49$, edad = 41,6 $\pm 10,9$ años) y obtenida en un estudio anterior (Reynaert, Janne et al. 1995c, Zdanowicz, Janne et al. 1998a), encontramos que son estadísticamente similares (Student's t-test $=2,0348, \mathrm{p}=0,945)$.

2. La "desresponsabilización" (retirada de la responsabilidad) de los otros (PHLC) es importante, puesto que es únicamente en términos de este parámetro, y a pesar de un incremento en la CHLC, el que un incremento en la puntuación relativa al factor "interno" se observa en términos de lo "interno"/"externo".

Pueden formularse tres hipótesis para explicar estos cambios:

- Que se dé un proceso originado en los adolescentes por el que renuncien a aceptar que otros deban continuar teniendo el derecho de intervenir en su salud.

- Que los mentores (padres) abandonen su responsabilidad paternalista y los adolescentes pasen a involucrarse más activamente en lo referente a su propia salud.

- O bien que, y esto es lo más probable, estos dos procesos tengan lugar concomitantemente.

3. Por último, resulta sorprendente observar cómo conforme los sujetos se hacen mayores tienden a pensar, de forma creciente, que el azar (CHLC) afecta al proceso de salud. Esto puede indicar cierto tipo de "reatribución": La creencia sobre el poder mágico conferido a los padres en su mítico papel de ser los protectores contra todo mal, se desplaza a partir de ese momento hacia el destino y la suerte...

\section{Conclusión}

Para concluir con estas observaciones, parece que este estudio puede tener implicaciones prácticas, como por ejemplo:

Podríamos reconsiderar nuestras estrategias en las campañas de prevención y dirigirlas no solamente a los adolescentes sino también a sus padres. Sería mejor que el mensaje dirigido hacia los adolescentes fuera indirecto, -destacando el hecho de que la salud ya no es asunto de sus padres-, más que directo (por ejemplo "la salud es asunto tuyo"). El mensaje dirigido a los padres debería invitarles a abandonar de forma paulatina su papel como supervisores de la salud.

Por la misma razón, podríamos preguntarnos si los resultados de la presente investigación pueden generalizarse a pacientes "no sanos". Por ejemplo, estos resultados darían a entender la inutilidad de tratar de persuadir a los pacientes crónicos en edad adolescente (desde la infancia) para que se involucraran más activamente en su tratamiento, siendo más juicioso sugerir que sus padres han dejado de ser los responsables. Al mismo tiempo, se debería pedir a los padres que deleguen la supervisión del tratamiento en sus hijos.

Las implicaciones en el caso de las enfermedades que se originan en la propia adolescencia son distintas. No existe evidencia de que la escala MHLC tenga características diferentes en adolescentes afectados por una enfermedad, en especial en el aspecto mental. Por ello, en un futuro estudio, trataremos de comparar la MHLC de nuestro grupo de jóvenes "sanos" con la de pacientes que sufren alguna enfermedad. También podría extenderse dicha comparación a aquellos sujetos que presentan conductas de riesgo. Aunque puede pensarse que tales sujetos presentaran una puntuación "interna" más 
baja que la de los sujetos "normales", tal suposición queda pendiente de confirmación.

\section{Bibliografía}

FONTAINE, P. Famille saines; esquisse conceptuelle générale. Thérapie familiale 3: 267-282, 1985.

JANNE, P. De la douleur à la plainte: pour une compréhension psychosomatique de l'ischémie myocardique dite silencieuse. Université Catholique de Louvain, Thèse de doctorat, Faculté de Psychologie et des Sciences de l'Education, 1989.

NADA-RAJA, S., MCGEE, R., WILLIAMS, S. Health beliefs among New Zealand adolescents. Journal of Paediatrics and Child Health 30: 523-529, 1994.

NORMAN, P., BENNATTE, P. Research and pratice with social cognition models in Conner, M., \& Norman, P. Predicting Health Behaviour. Bristol: The open University, 1996.

OLSON, D.H., SPRENKLE, D.H., RUSSELL, C.S. Circumplex model of marital and family systems: Cohesion and adaptability dimensions, family types and clinical applications. Family Process 12: 179-188, 1973.

OLSON, D.H., KLLLORIN, E. Clinical rating scale for circumplex model (revised version), St Paul MN: Family Social Science: University of Minnesota Press, 1985.

OLSON, D.H. Circumplex model: validation studies FACES III. Family Process 25: 337-351, 1986.

OLSON, D.H. Tree dimensional (3-D) circumplex model: theoretical and methodological advances. Minnesota: University of Minnesota Press, 1991.

Organisation Mondial de la santé (WHO). La santé des jeunes, Genève: OMS, 1994.

PAUWELS, A., JANNE, P., REYNAERT, CH. De différents modèles de croyances envers la santé au vécu subjéctif de contrôle vis à vis de la santé: une tentative d'approche intégrative. Journal de thérapie comportementale et cognitive 9: 99-107, 1999.

REYNAERT, CH., JANNE, P., DONCKIER, J., BUYSSCHAERT, M., ZDANOWICZ, N., LEJEUNE, D., CASSIERS, L. Locus of control and metabolic control. Diabète et Métabolisme 21: 180-187, 1995a.

REYNAERT, CH., JANNE, P., DELIRE, V., PIRARD, M., RANDOUR, PH., COLLARD, E., INSTALLE, E.,
COCHE, E., CASSIERS, L. To control or to be controlled? From health locus of control to morphine during patientcontrolled analgesia. Psychotherapy and Psychosomatics 319: 1-8, 1995b.

REYNAERT, CH., JANNE, P., BOSLY, A., STAQUET, P., ZDANOWICZ, N., VAUSE, M., CHATELAIN, B., LEJEUNE, D. From health locus of control to immune control: internal locus of control has a buffering effect on natural killer cell activity decrease in major depression. Acta Psychiatrica Scandinavia 92: 294-300, 1995c.

SLOMOWITZ, M. Adolescent psychoterapy. Washington: American Psychiartic Press, 1991.

STANTON, W.R., NADA-RAJA, S., LANGLEY, J. Stability in the structure of health locus of control among adolescents. British Journal of Clinical Psychology, 34: 279-287, 1995.

STUBHER, M.L. Psychiatric aspects of organ transplantation in children and adolescents. Psychosomatics 34: 379-389, 1993.

WALLSTON, K.A., WALLSTON, B.S., DE VELLIS, R. Development of the multidimensional health locus of control (MHLC) scale. Health Education Monagraphy, 6: 160-170, 1978.

WALLSTON, K.A., WALLSTON, B.S., SMITH, M.S., DOBBINS, C.J. Perceived control and health', Current Psychology Research and Revue 6: 5-25, 1987.

ZDANOWICZ, N., REYNAERT, CH., DELAUNOIS, L., JANNE, P. Complications Psychiatriques des greffes d'organes. Annales Médico-Psychologiques 154: 232-237, 1996.

ZDANOWICZ, N., JANNE, P., REYNAERT, CH., ROUSSAUX, J-P. The dicrepancy between alcoholics' perceived control and actual skills. European Journal of Psychiatry 12: 205-221, 1998a.

ZDANOWICZ, N., REYNAERT, CH., JANNE, P., INSTALLE, E., EVERARD, P., DELAUNOIS, L. Transplants and Psychiatry. Psychosomatics 39: 390-391, 1998 b.

Dirección para correspondencia:

N. Zdanowicz

Universidad Católica de Lovaina

Clínica de Mont-Godinne

Unidad de Psicosomática

5530 Yvoir

E-mail: nicolas.zdanowicz@pscl.ud.ac.be

BÉLGICA 